\title{
Actividad anti fúngica (in vitro) de extractos vegetales para el control de antracnosis
}

\author{
(Colletotrichum acutatum)
}

\author{
Antifungal (in vitro) activity of plant extracts for the control of anthracnose
}

\section{(Colletotrichum acutatum)}

Villacís-Aldaz Luis Alfredo*, León-Gordon Olguer, Santana-Mayorga Rita, Mangui-Tobar José, Carranza Galo, Pazmiño-Miranda Pilar

\begin{tabular}{l}
\hline Datos del Artículo \\
\hline Facultad de Ciencias Agropecuarias \\
Universidad Técnica de Ambato, Tungu- \\
rahua, Ecuador. \\
Casilla postal: 18-01-334. \\
Telf: (+593)032872630-0985471191. \\
ao.leon@uta.edu.ec \\
rc.santana @uta.edu.ec \\
jmanguitobar@yahoo.es \\
gcarranza5672@uta.edu.ec \\
nd.pazmino@uta.edu.ec \\
*Dirección de contacto: \\
Luis Alfredo Villacís Aldaz \\
Facultad de Ciencias Agropecuarias. \\
Universidad Técnica de Ambato. \\
Tungurahua, Ecuador. \\
Casilla postal: $18-01-334$. \\
Telf: (593) 032872630 - 0985471191 \\
E-mail: la.villacis@uta.edu.ec \\
\hline \\
\hline Palabras clave: \\
Micelial, \\
in vitro, \\
inhibición, \\
patógeno, \\
maceración. \\
\end{tabular}

J Selva Andina Biosph. 2017; 5(1):59-64.

\section{Historial del artículo.}

Recibido septiembre, 2016.

Devuelto enero 2017

Aceptado marzo, 2017.

Disponible en línea, mayo 2017.

Editado por:

Selva Andina

Research Society

\section{$\underline{\text { Key words: }}$}

Micelial,

in vitro,

inhibition,

pathogen,

maceration.

\section{Resumen}

Se evaluó el efecto anti fúngico de la aplicación de cinco extractos vegetales: ortiga (Urtica dioica), manzanilla (Chamaemelum nobile), marco (Artemisia vulgaris), lavanda (Lavandula officinalis) y chamico (Datura ferox), a nivel de laboratorio para control de antracnosis (Colletotrichum acutatum) del tomate de árbol (Solanum betaceum), utilizando dos métodos de extracción (maceración y arrastre de vapor), en los resultados obtenidos, se observa diferencias estadística en el porcentaje de inhibición de crecimiento micelial del hongo, el tratamiento con extracto de lavanda obtenido por el método de arrastre de vapor, presentó la mayor eficacia para control de antracnosis (66.23\%). Los demás tratamientos ensayados también presentó actividad contra el patógeno de la antracnosis, en el siguiente orden de inhibición: manzani1la $(52.78 \%)$, marco (21.63), chamico (24.14\%), ortiga (12.94), la capacidad de diversas especies de inhibir ciertas enfermedades fúngicas, asumiéndose al contenido diferente de metabolitos secundarios que tienen cada una de las especies vegetales, constituyendo en una alternativa de producción limpia, que reduzcan la utilización de pesticidas químicos para el control de las enfermedades fúngicas.

(C) 2017. Journal of the Selva Andina Biosph. Bolivia. Todos los derechos reservados.

\section{Abstract}

The antifungal effect of five plant extracts: nettle (Urtica dioica), chamomile (Chamaemelum nobile), frame (Artemisia vulgaris), lavender (Lavandula officinalis) and chamico (Datura ferox) were evaluated at laboratory level for control of anthracnose (Colletotrichum acutatum) of the tree tomato (Solanum betaceum), using two methods of extraction (maceration and steam trapping), in the results obtained, statistical differences were observed in the percentage of inhibition of mycelial growth of the fungus, the treatment with lavender extract obtained by the steam trapping method presented the greatest efficiency for anthracnose control (66.23\%). The other treatments tested also showed effectiveness against the anthracnose pathogen in the following order of inhibition: chamomile (52.78\%), frame (21.63), chamico (24.14\%), nettle (12.94), the ability of various species to inhibit certain fungal diseases, taking into account the different content of secondary metabolites that have each of the plant species, constituting new clean production alternatives that reduce the use of chemical pesticides for the control of fungal diseases. 


\section{Introducción}

La enfermedad común en frutos de tomate de árbol (Solanum betaceum Cav.), es la antracnosis (Colletotrichum acutatum), causante de significativas pérdidas económica para los agricultores de la zona central del Ecuador, siendo el principal método de control los agroquímicos (Bolívar et al. 2009). El excesivo uso de pesticidas sintéticos que se viene utilizando en la explotación agrícola del tomate de árbol, hace que los productos que consumimos a diario en nuestra alimentación se encuentren contaminados por las altas dosis de pesticidas, lo cual está afectando la salud de los seres humanos y el deterioro del medio ambiente (Alvarez et al. 2011) . Pérez \& Iannacone 2006, manifiestan que la selección de la especie de planta es el primer paso dentro de la investigación de nuevos plaguicidas botánicos. Estos bioensayos se emplean para estudiar las propiedades biocidas de las diferentes partes de las plantas (raíces, corteza, hojas, frutos, flores, etc.), la eficacia de los diferentes extractos y formulaciones, y el modo de acción de los ingredientes activos. Los bioensayos deben ser altamente sensitivos a las sustancias bioactivas, fáciles de manipular, baratos, de amplio espectro, y dar rápidos resultados. Además que para el desarrollo tecnológico de un plaguicida botánico se deben estandarizar sus métodos de extracción y la propagación de las plantas.

La utilización de insumos agrícolas en la agricultura se ha extendido, considerados esenciales para lograr mejores condiciones de cultivo. Sin embargo, la lista de sustancias químicas utilizadas incluye algunas peligrosas con dosis cada vez más altas, creando resistencia a ciertas enfermedades como la antracnosis (Villacís-Aldaz et al. 2016), la problemática descrita ha conllevado a organizaciones como la FAO y la Organización Mundial de la Salud (OMS) a insistir en la necesidad de adoptar medidas de precaución en su manipulación, aplicación, para así tener productos limpios sin residuos químicos (Centurión-Belotto et al. 2013).

La necesidad de buscar alternativas naturales para el control de antracnosis en tomate de árbol y comenzar a prescindir de los pesticidas sintéticos, siendo los extractos botánicos que ofrecen seguridad para el medio ambiente y una eficiente opción agronómica (Briceño et al. 2011). Investigaciones realizadas (Stauffer et al. 2000, Rodríguez \& Sanabria 2005, Maselli et al. 2008, Pino et al. 2008) manifiestan que los extractos botánicos pueden ser utilizados exitosamente en el control o inhibición de bacterias y hongos fitopatógenos, además pueden constituirse en una herramienta para integrar a un manejo agroecológico encaminada a una agricultura sustentable.

En busca de nuevas técnicas de producción para los agricultores, se planteó evaluar la actividad antifúngica de los extractos vegetales (EV) para el control in vitro de Colletotrichum acutatum aislado de tomate de árbol (Solanum betaceum Cav.).

\section{Materiales y métodos}

La presente investigación se llevó a cabo en el Laboratorio de Fitopatología de la Facultad de Ciencias Agropecuarias de la Universidad Técnica de Ambato, Tungurahua-Ecuador, bajo condiciones controladas de humedad y Temperatura, realizando los experimentos in vitro.

Para la preparación de los extractos vegetales se recolectaron de la zona central Andina material vegetal, con posibles propiedades antifúngicas de: ortiga (Urtica dioica), manzanilla (Chamaemelum nobile), marco (Artemisia vulgaris), Lavanda (La- 
vandula officinalis) y chamico (Datura ferox). Se utilizó el equipo de destilación por arrastre de vapor (Clevenger) para obtener los extractos botánicos de cada una de las plantas en relación de $1 \mathrm{~kg}$ de hojas fresca en $1 \mathrm{~L}$ de agua, La segunda metodología de extracción consistió en la maceración en condiciones de laboratorio a una temperatura de $15{ }^{\circ} \mathrm{C}$, donde el material vegetal se lo dejo secar a la sombra por $72 \mathrm{~h}$, procediendo a pulverizarlo en un molino de martillos, se mezcló $50 \mathrm{~g}$ en $30 \mathrm{~mL}$ de alcohol etílico al 70\%, dejándolo macerar por 4 días, tiempo en el cual se procede a filtrar y a concentrar a baño maría hasta obtener el $20 \%$ del volumen inicial, de cual se aplicó $1 \mathrm{~mL}$ en cada uno de los tratamientos. Para el aislamiento del agente causal de la antracnosis, se tomaron muestras del hongo fitopatógeno de las hojas y frutos del tomate de árbol, llevándolo a una cámara húmeda, con temperatura ambiente hasta que fructifique, para luego sembrar las esporas en cajas Petri que contienen medio de cultivo (Agar papa dextrosa), incubando a $28{ }^{\circ} \mathrm{C}$ y humedad del $70 \%$.

Tabla 1 Descripción de los tratamientos

\begin{tabular}{ccl}
\hline No & Tratamientos & \\
\hline T1 & E1M1 & Extracto vegetal de manzanilla (Matricaria recutita) por hidrodestilación \\
T2 & E1M2 & Extracto vegetal de manzanilla (Matricaria recutita) por maceración \\
T3 & E2M1 & Extracto vegetal de chamico (Datura metel) por hidrodestilación \\
T4 & E2M2 & Extracto vegetal de chamico (Datura metel) por maceración \\
T5 & E3M1 & Extracto vegetal de marco (Ambrosia arborescens ) por hidrodestilación \\
T6 & E3M2 & Extracto vegetal de marco (Ambrosia arborescens) por maceración \\
T7 & E4M1 & Extracto vegetal de lavanda (Lavandula officinalis) por hidrodestilación \\
T8 & E4M2 & Extracto vegetal de lavanda (Lavandula officinalis) por maceración \\
T9 & E5M1 & Extracto vegetal de ortiga (Urtica dioica) por hidrodestilación \\
T10 & E5M2 & Extracto vegetal de ortiga (Urtica dioica) por maceración \\
T11 & Testigo & \\
\hline
\end{tabular}

Para el análisis de los tratamientos (Tabla 1), se utilizó el diseño experimental de bloques completos al azar con arreglo factorial de $5 \times 2+1$ con tres repeticiones. Los datos obtenidos fueron sometidos a análisis de varianza (ANOVA), con las respectivas pruebas de significación Tukey al 5\%, utilizando el software Statistix 9.

Las variables respuesta fueron:

Porcentaje de inhibición determinada según la fórmula propuesta por (Martínez et al. 1996).

$$
\% \text { Inhibición }=\left(1-\frac{\text { Crecimiento tratamiento }(\mathrm{mm})}{\text { Crecimiento testigo }(\mathrm{mm})}\right) * 100
$$

La velocidad de crecimiento radial, se la registro a $\operatorname{los} 48,96$ y $144 \mathrm{~h}$ de haber aplicado el tratamiento correspondiente, utilizando la relación de ArangoBedoya et al. (2015).

$$
V C R(\mathrm{~mm} / \mathrm{d})=\frac{\text { Crecimiento Radial }(\mathrm{mm})}{\text { Número de Días }}
$$

El registró de la viabilidad de los conidios, observándose con la ayuda de la cámara de Neubauer y el estereoscopio, contabilizando los conidios que aún estaban en la fase germinación y los no viables. 


\section{Resultados}

Para la variable porcentaje de inhibición micelial, los resultados fueron estadísticamente significativos ( $\mathrm{p}<0.01)$, tanto a las 48, 96 y $144 \mathrm{~h}$ de aplicado los tratamientos, destacándose los tratamientos $\mathrm{T} 7$ y T8, correspondiente a los extractos de lavanda, (Figura 1) obtenido por arrastre de vapor y maceración respectivamente, seguido se observaron a los extractos de manzanilla (T1 y T2) que se ubicaron en el segundo rango de la prueba de significación de Tukey al 5\% (Figura 2), los demás tratamientos se ubicaron y compartieron rangos inferiores, demostrando menor inhibición micelial: manzanilla (52.78\%), marco (21.63), chamico (24.14\%), ortiga (12.94). En las demás variables respuesta analizadas se mantuvieron similares tendencias al de porcentaje de inhibición.

Figura 1 Aplicación del extracto de lavanda sobre micelios de antracnosis a las 24 horas
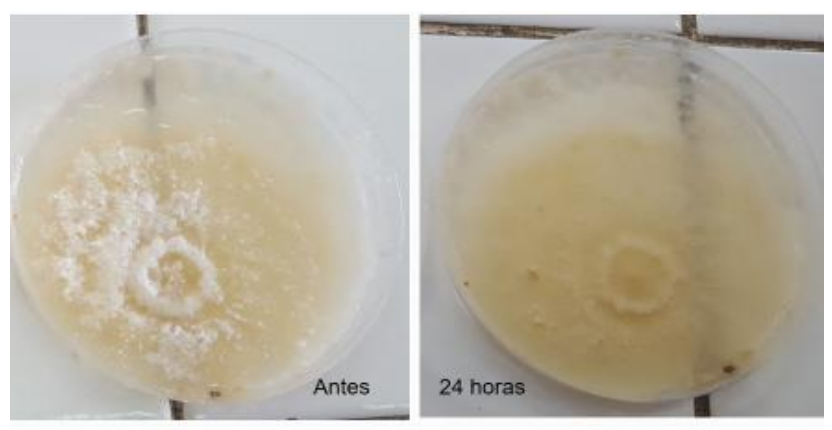

En el análisis de varianza para las variables: Velocidad de crecimiento radial y viabilidad de conidios, reportaron alta significación $(\mathrm{p}<0.01)$ entre los tratamientos, observando a la lavanda, manzanilla y marco, con los mejores resultados para el control de antracnosis. Cabe mencionar que entre los dos métodos de obtención de los extractos vegetales, se encontró diferencias estadísticas significativas, resultando el equipo de arrastre de vapor con mayor eficiencia a la hora de obtener metabolitos secundarios principalmente.

Figura 2 Comparación de medias para la variable porcentaje de inhibición micelial a las 144 horas de aplicado el tratamiento

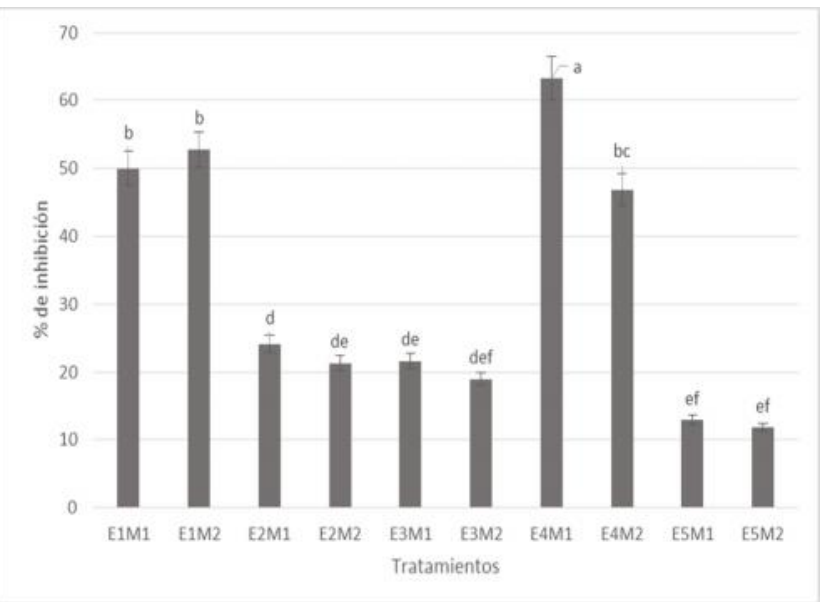

\section{Discusión}

Los extractos vegetales aplicados en la presente investigación causaron sensibilidad in vitro diferente para el hongo $C$. acutatum, lo que podría ser debido al contenido de los aceites esenciales de cada uno de los extractos vegetales aplicados (Bolívar et al. 2009), de manera general están constituidos de compuestos terpénicos y fenólicos, capaces de a afectar la permeabilidad de las membranas, provocando trastornos metabólicos inhibición el crecimiento micelial (Gende et al. 2008).

En el extracto de lavanda (63.27\% de inhibición), se observó un porcentaje alto de control, en relación a los demás tratamientos, deduciendo probablemente al contenido de los metalitos secundarios como el linalol (30-40\%) y acetato de linalilo (35-55\%) que lo constituye, lo que concuerda con Montes et al. (1992).

Los demás tratamientos ensayados también demostraron efectividad contra el patógeno de la antracno- 
sis a nivel laboratorio, en el siguiente orden de inhibición: manzanilla, marco, chamico, ortiga, lo que corrobora con otros trabajos de investigación, demostrado la capacidad de diversas especies de inhibir de ciertas enfermedades fúngicas, asumiendo a la presencia de los metabolitos secundarios, donde sus compuestos fenólicos junto con los alcaloides, son de amplio interés en la bioquímica, por cuanto están presentes en las plantas superiores, específicamente en los órganos vegetales (Briceño et al. 2011).

Con respecto a las diferentes metodologías de obtención de los extractos vegetales, en los tratamientos analizados, el arrastre de vapor, es superior al de maceración, en donde los aceites esenciales tienen mayor concentración y rendimiento, ante lo cual, la mencionada técnica está siendo utilizada de forma habitual a nivel de laboratorio (Cerutti \& Neumayer 2004).

Se concluye que a nivel de laboratorio in vitro, los extractos vegetales estudiados tienen diferentes propiedades anti fúngicas para el control de antracnosis (Colletotrichum acutatum), que pueden constituir en nuevas alternativas de producción agroecológicas, que reduzcan la utilización de pesticidas químicos para el control de las enfermedades fúngicas, sugiriendo realizar estudios a nivel de campo con los extractos vegetales que dieron mejores resultados, permitiendo obtener funguicidas biológicos que sean distribuidos a los agricultores.

\section{Conflictos de intereses}

Esta investigación se la realizó en la Universidad Técnica de Ambato, Facultad de Ciencias Agropecuarias, Tungurahua Ecuador y no presenta conflictos de interés.

\section{Agradecimientos}

Los autores agradecen a la Universidad Técnica de Ambato Facultad de Ciencias, por el apoyo técnico, científico y logístico realizado a la presente investigación.

\section{Literatura citada}

Alvarez-S DE, Salazar-G CE, Hurtado-B AM, Delgado-B DM, Arango-B O. Sensibilidad in vitro de phytophtora infestans al extracto de fique (Furcraea gigantea Vent.) y fungicidas sistémicos. Rev Bio Agro 2011;9(2):96-104.

Arango-Bedoya Ó, Hurtado-Benavides AM, Pantoja-Daza D, Santacruz-Chazatar L. Actividad inhibitoria del aceite esencial de Lippia origanoides H.B.K sobre el crecimiento de Phytophthora infestans. Acta Agron 2015;64(2):11624.

Bolívar K, Sanabria ME, Rodríguez D, Camacaro M, Ulacio D, Cumana L, et al. Potencial efecto fungicida de extractos vegetales en el desarrollo in vitro del hongo Colletotrichum gloeosporioides (Penz.) Penz. \& Sacc. y de la antracnosis en frutos de mango. Revista UDO Agrícola 2009;9(1):175-82.

Briceño G, García J, Maselli A, Rosales LC. Efecto de extractos etanólicos de ruda y nim sobre el control de bacterias fitopatógenas del género "Erwinia". Agron Trop 2011;61(2):141-8.

Centurión-Belotto SA, Aquino-Jara AS, BozzanoSaquier G. Extractos vegetales para el control alternativo del damping-off causado por Rhizoctonia solani en plántulas de tomate. Investig Agrar 2013;15(1):23-9. 
Cerutti M, Neumayer F. Introducción a la obtención de aceite esencial de limón. Invenio 2004; 7(22):149-55.

Gende LB, Principal J, Maggi MD, Palacios SM, Fritz R, Eguaras MJ, et al.. Extracto de Melia azedarach y aceites esenciales de Cinnamomun zeylanicum, Mentha piperita y Lavandula officinalis como control de Paenibacillus larvae. Zootecnia Trop 2008;26(2):151-6.

Martínez MJ, Betancourt-Badell J, Alonso-Gonzá lez N. Ausencia de actividad antimicrobiana de un extracto acuoso liofilizado de Aloe vera (Sábila). Rev Cubana Plant Med 1996;1(3):1820.

Maselli A, Méndez R, García J, Briceño G, Solano A, Alemán L, et al. Evaluación de extractos vegetales para el control de bacterias fitopatógenas de los géneros Xanthomonas y Erwinia. Fitosanidad 2008;12(3):164.

Montes M, Wilkomirsky T, Valenzuela L. Plantas medicinales. Universidad de Concepción, Concepción, Chile 1992; p. 207.

Pérez D, Iannacone J. Efectividad de extractos botánicos de diez plantas sobre la mortalidad y repelencia de larvas de Rhynchophorus palmarum L., insecto plaga del Pijuayo Bactris gasipaes Kunth en la Amazonía del Perú. Agric Téc 2006;66(1): 21-30.
Pino O, Jorge-Lazo F, Sánchez Y, Iglesias A, García L, Khambay BPS. Extractos naturales y bioplaguicídas para el control de plagas. Fitosanidad 2008;12(3):163.

Rodríguez DA, Sanabria ME. Efecto del extracto de tres plantas silvestres sobre la rizoctoniosis, la mancha sureña del maíz y los patógenos que las causan. Interciencia 2005;30(12):739-44.

Statistix for Windows version 9.0. User's Manual. Analytical Software. Tallahassee, FL, USA. Statistix.

Stauffer BA, Orrego A, Aquino A. Selección de extractos vegetales con efecto fungicida y/o bactericida. Rev Cienc Tecnol 2000;1(2):2933.

Villacís-Aldaz LA, Zapata-Vela JJ, León-Gordón OA, Vásquez-Freitez CL, Mullo-Sarzosa JG, Zapata-Vela AC, et al. Compatibilidad y sobrevivencia de microorganismos benéficos de uso agrícola (Beauveria bassiana, Bacillus thuringiensis y Paecilomyces lilacinus) en compost. J Selva Andina Biosph 2016;4(2):939. 